\title{
Digital Image Modification Detection using Color Information and its Histograms
}

\author{
Anonymous submission for blind review
}

\begin{abstract}
The rapid development of many open source and commercial image editing software makes the authenticity of the digital images questionable. Copy-move forgery is one of the most widely used tampering techniques to conceal undesirable objects in a scene. Existing techniques reported in the literature to detect such tampering aim to improve the robustness of these methods against the use of JPEG compression, blurring, noise, or other types of post processing operations. These post processing operations are frequently used with the intention to conceal tampering and reduce tampering clues. A robust method based on the color moments and other five image descriptors is proposed in this paper. The method divides the image into fixed size overlapping blocks. First color moments of the blocks are used to group similar blocks into clusters. Clustering operation divides entire search space into smaller pieces with similar color distribution. Blocks from the tampered regions will reside within the same cluster since both copied and moved regions have similar color distributions. Five image descriptors are used to extract block features, which makes the method more robust to post processing operations. An ensemble of deep compositional pattern-producing neural networks are trained with these extracted features. Similarity among feature vectors in clusters indicates possible forged regions. Experimental results show that the proposed method can detect copy-move forgery even if an image was distorted by gamma correction, addictive white Gaussian noise, JPEG compression, or blurring.
\end{abstract}

Keywords: Image tampering techniques, image feature extractor, copy-paste forgery, digital forensics

\section{Introduction}

Image modification may be a strong indication of digital forgeries, which have attracts great intention of the forensic science research community in the recent years $[5,6,7]$. There are several types of digital image tampering techniques and copy move forgery is the most common technique. It is either used to conceal or to duplicate an object in the image. A carefully selected portion of the image is copied and pasted onto another different location in the same image. Some post processing operations such as JPEG compression, noise adding or Gaussian blurring can be applied to the forged image to hide the clues about forgery. However, copied and pasted regions have similar features even if they were post processed to an extent. Human eyes cannot perceive forgery operation easily. 
Researchers proposed many techniques to detect copy move forgery. Most techniques seek a global similarity among local regions. Suspicious image is partitioned into overlapping blocks and various feature extraction methods are used to represent these blocks. Then, features are lexicographically sorted to move similar blocks closer and a similarity comparison is performed over a window of feature vectors to find the duplicated regions. Some of the differences between these methods are from the perspectives of their feature extraction methods, their geometry of the blocks (rectangular or circular), their feature vector size and their sorting algorithms to move similar vectors closer together.

DCT (Discrete Cosine Transform) based forgery detection methods used the DCT coefficients to represent the overlapping blocks. First method to detect the copy move forgery proposed by the Fridrich et al. was based on the DCT [2]. Their method divides the image into overlapping blocks. DCT coefficients of each block constitute the feature vectors. Feature vectors are stored in a matrix and then lexicographically sorted. Similarity comparison between adjacent vectors in the matrix is performed to detect forged regions. After this work, Wang et al. used DCT and DWT (Discrete Wavelet Transform) together to improve the robustness of the method against JPEG compression and additive noise [3]. The method applied DWT and DCT on the overlapping image blocks. Coefficients obtained by these transformations were multiplied to form the eigenvectors. Mean and variance of the distances between the eigenvalues in their corresponding eigenvectors are used to decide the similarity between two blocks. In 2011, Hu et al. used grouped DCT coefficients to form the feature vectors [4]. Their algorithm sorts the distance between every pair of DCT-coefficient formed vectors instead of the feature vectors. If the distance between two blocks is less than a threshold, two blocks will be assumed to be similar. The algorithm is robust to noise addition, but will fail to detect manipulation when other image processing operations are used to conceal the forgery. Huang et al. aimed to improve the speed of the processing of Fridrich et al.'s work [5]. Their work quantized the DCT coefficients with predefined value $q$. Their method used a new method to decide the similarity between adjacent feature vectors. Results show that their method is robust to some post processing operations. Cao et al. used the mean of the DCT coefficients to reduce the dimension of the feature vectors [6]. Their method gains robustness against Gaussian distributed additive noise post processing operations since the mean of the DCT coefficients are less sensitive to processing. The feature vector dimension proposed by their method is $1 \mathrm{x} 4$. Euclidean distance between the vectors is used as a clue about possible forgery. Two blocks are considered to be identical if the corresponding distance is less than a predefined threshold. Zhao et al. proposed a method based on DCT and SVD (Singular Value Decomposition) in 2013 [7]. The method applied DCT on each overlapping blocks. DCT coefficients are quantized by predefined matrix to obtain a more robust representation. Each quantized block is divided into non-overlapping blocks and SVD is applied to each sub-block. Experimental results show that the method can detect forgery even when forged image is postprocessed. 
Myna et al. used Log-polar transform to detect the forgery in their work [8]. Their method used DWT to reduce image size. Using Log-polar transform for feature extraction makes the method robust against rotation, scaling and translation attacks. Bayram et al. used the Fourier Mellin Transform in their work [9]. They used bloom filters to reduce the computational complexity of the block-matching phase. Popescu and Farid used PCA in copy move detection to detect the forgery [10]. They truncated less significant eigenvectors to ensure higher discriminative power. Zimba and Xinging used DWT to reduce image size [11]. Thus, their work improved Popescu et al.'s work by using smaller feature vectors. Li et al. used DWT and SVD together to detect the copy move forgery [12]. Ting and Rang-Ding utilized SVD with KD-tree in their work [13]. Some authors also used invariant image moments to generate feature vectors $[14,15]$. The main advantage of using image moments is their robustness to some deformations such as geometric operations. Luo used block intensity ratio and color features to ensure the robustness of their method. Mahdian et al. used blur moment invariants to make the method more robust against blurring operations.

Long and Huang [23-25] modeled the problem using a quadratic form and discovered that, scene correlation generally distributes evenly over Principal Components Analysis (PCA) space of the quadratic coefficient matrix while demosaicking inter-pixel correlation clusters at dimensions corresponding to large eigenvalues. If the quadratic coefficient matrix is considered as features, this model is appropriate to be viewed as feature-based. Huang and Fan [26] proposed to used compositional pattern-producing networks for image forgery detection and digital forensics.

A method based on color moments and other five image descriptors is proposed in this paper to improve the robustness of the DCT based methods against some post processing operations, i.e. JPEG compression, Gaussian blurring and additive white Gaussian noise. The method divides input image into overlapping blocks. The first color moments for each of the three color channels are calculated and blocks are grouped into thirteen clusters according to their color moments. Blocks that have similar color distribution are grouped into the same cluster. Then, feature vectors for blocks in each cluster are extracted concurrently by separate threads to speed up the process. The color layout descriptor algorithm is used for feature extraction [16]. Each thread concurrently creates a matrix of feature vectors and evaluates similarity among them for possible forgery. If the number of blocks with similar feature vectors exceeds a predefined threshold value, corresponding blocks are marked as forged by the method. Experimental results show that the method has higher accuracy ratio compared to other DCT based algorithms even after post processing.

The rest of the paper is organized as follows. Technical details of the color moments are other five image descriptors are reviewed in Section 2. We present our proposed method in Section 3. The experimental results are provided and discussed in Section 4. Finally Section 5 contains some concluding remarks. 


\section{Review of Image Descriptors}

In this section, we give the technical details of the color moments, color layout descriptors, color and edge directivity descriptor, fuzzy color and texture histogram, scalable color descriptor, and edge histogram descriptor. The proposed method uses these techniques to detect copied and moved regions in images.

\subsection{Color Moments}

Content-Based Image Retrieval (CBIR) systems have become a popular topic among researchers recently. These systems permits the users to search and browse through image and video databases. CBIR systems use different feature extraction algorithms to query an image database for a candidate. Many image retrieval systems use visual contents such as color, texture and shape to retrieve all the images whose contents are similar to the visual contents of the query image. Color is one of the most widely used features to discriminate pictures for image retrieval. Color histograms that are resistant to position and orientation changes, are used by many methods to filter the image database. However, spatial relationship of color regions cannot be used by the histograms to discriminate the images. Color correlogram and color coherence vectors also use global distribution of local colors. These methods yield better results compared to color histograms for image retrieval. But computational complexity is the most important disadvantage of these methods. Stricker et al. showed that color moments could be used to characterize the color distribution of the images [17]. First three moments of the three channels are measures that can be used differentiate images based on their color features. These moments can be used to assess color similarity between images.

First color moment selected is the average color intensity of the image. Square root of the standard deviation is selected as the second color moment. Third moment gives an idea about the degree of asymmetry in the distribution. The proposed method uses first color moments to cluster sub-blocks according to their color distribution.

\subsection{Color Layout Descriptor}

The purpose of the MPEG-7 standard is to provide standardized descriptions of streamed or stored images [16]. Thus, the users or applications can categorize images or videos. Visual descriptions supply non-textual information to compare, filter or browse images or videos. MPEG-7 visual feature descriptors provide the users capability to query an image database with a query image. Additionally, action based searching in the video database can be realized using visual descriptors. Color Layout Descriptor (CLD) is one of the visual descriptors of the MPEG-7 standard.

The CLD represents the spatial distribution of colors in the image. It supports high-speed image retrieval and represents color resolution invariant. Many techniques such as similarity based retrieval; content filtering can use this descriptor for feature extraction. The algorithm to compute the CLD 
vector from an image consists of four steps. The image is partitioned first, and then a representative color is selected. Then, the DCT transform is applied and a zigzag scanning is performed as the last step. The image is converted into $\mathrm{YCbCr}$ color space, before CLD feature extraction. The detail of the algorithm is given below.

CLD algorithm uses a selection algorithm to represent the sub-block with a representative color. Standard uses the average of pixels in a sub-block as the corresponding representative color. Representative colors for each block constitutes an $8 \times 8$ tiny image of the input image. The color space conversion from $\mathrm{RGB}$ to $\mathrm{YCbCr}$ is realized on the tiny image. The three channels of the tiny image $(\mathrm{Y}, \mathrm{Cb}, \mathrm{Cr})$ are transformed into frequency domain using the DCT. Then a zigzag scanning is performed on DCT coefficient matrices to form three vectors of size $1 \times 64$, which is the CLD of the image. The proposed method uses CLD during feature extraction from the sub-blocks of the image.

\subsection{Color and Edge Directivity Descriptor}

Color and Edge Directivity Descriptor (CEDD) extracts the color information from regions of the image using a set of fuzzy rules and resulting in a HSV color space histogram. It includes texture information using the proposed MPEG-7 [1] Edge Histogram Descriptor rules. Finally, it uses Gustafson Kessel fuzzy classifier [19] to binarize the histogram. For the implementation of this feature, LIRe was used [20].

\subsection{Fuzzy Color and Texture Histogram}

Very similar to the CEDD feature, FCTH mainly differs in using Haar Wavelet transform to model texture information. For the implementation of this feature, LIRe was used. [21].

\subsection{Scalable Color Descriptor}

The Scalable Color Descriptor is derived from a color histogram defined in the Hue SaturationValue (HSV) color space with fixed color space quantization. It uses a Haar transform coefficient encoding, allowing scalable representation of description, as well as complexity scalability of feature extraction and matching procedures.

\subsection{Edge Histogram Descriptor}

The Edge Histogram Descriptor (EHD) basically represents the distribution of 5 types of edges in each local area called a sub-image [22]. The sub-image is defined by dividing the image space into $4 \times 4$ non-overlapping blocks. Thus, the image partition always yields 16 equal-sized sub-images regardless of the size of the original image. To characterize the sub-image, we then generate a histogram of edge distribution for each sub-image. Edges in the sub-images are categorized into 5 types: vertical, horizontal, 45-degree diagonal, 135-degree diagonal and non-directional edges (refer 
to Figure 1). Thus, the histogram for each sub-image represents the relative frequency of occurrence of the 5 types of edges in the corresponding sub-image. As a result, each local histogram contains 5 bins. Each bin corresponds to one of 5 edge types. Since there are 16 sub-images in the image, a total of $5 \times 16=80$ histogram bins is required (see Figure 2). Note that each of the 80 - histogram bins has its own semantics in terms of location and edge type.

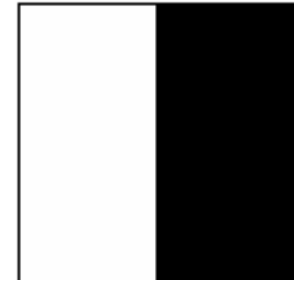

a) vertical edge

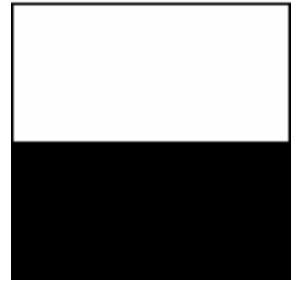

b) horizontal edge

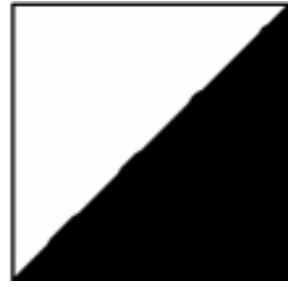

(c) 45-degree edge

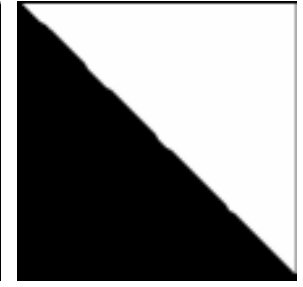

(d) 135-degree edge (e) non-directional edge

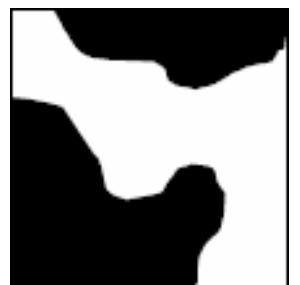

Figure 1. Five genres of image edges
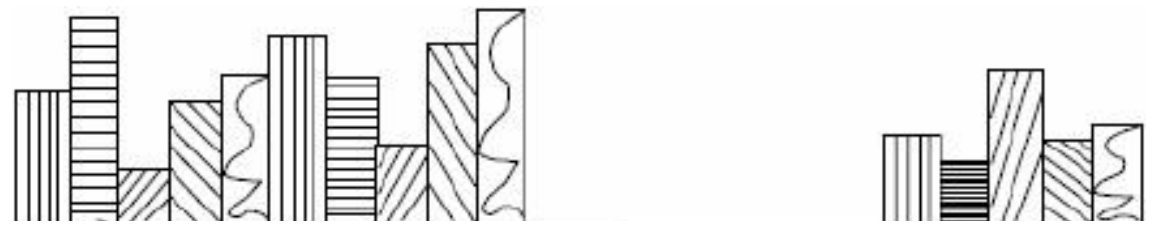

Figure 2. 1-D array of 80 bines of the EHD

\section{The Proposed Method}

Many forged images have at least two similar regions in order not to change the global statistics of the image to minimize the probability of detection. However, a natural image unlikely contains two similar regions other than large smooth regions such as the sky. The task of finding copied and pasted regions in an image is to search similar regions in an image. If an algorithm implements the pixel-by-pixel comparison to find every similar pairs, computational complexity of the algorithm may be very high. So Fridrich et al. [2] suggested that the suspicious image must be divided into overlapping blocks to decrease the processing time and to find the similar regions in a reasonable computational load.

Most frameworks for forgery detection algorithms in the existing literature use the same steps during the detection phase for feature extraction from the image blocks. A good feature extraction method ensures that the algorithm gives comparable results even if the forged image is postprocessed. Thus, capability of the feature extraction method determines accuracy ratio of a forgery detection method.

Many methods in the existing literature follow the similar steps: Divide the image into overlapping blocks, represent each block with a feature vector, create a matrix from feature vectors, sort the matrix lexicographically, compare every vector in the matrix with local neighborhood and 
decide similarity, mark blocks as forged using similarity between vectors as the indication of the forgery.

Detailed description of the proposed detection algorithm is given below:

In the proposed method, original image is divided into overlapping fixed sized sub blocks as the first step. Blocks are clustered according to their color similarity using color moments during the second step. Similarity between feature vectors of the sub-blocks is accepted as a clue about possible forgery.

Assume that the suspicious image is a color image I of size $M$ by $N$. The algorithm splits input image into overlapping blocks of size $L$ by $L$. Each block is denoted as $L_{i j}$ where $i, j$ denotes upper left coordinates starting point of the corresponding block. Sliding an $L x L$ window one pixel each step from the upper left corner right down to the lower right corner defines all overlapping blocks. The sliding process will generate $(M-L+1) \times(N-L+1)$ blocks.

For each image block, the algorithm extracts their color moments, color layout descriptors, color and edge directivity descriptor, fuzzy color and texture histogram, scalable color descriptor, and edge histogram descriptor, respectively, according to the description in Section 2. These extracted features form a 50-dimensional feature vector for each image block, which is fed to a Compositional PatternProducing Network (CPPNs) for deep learning purpose. CPPNs are a variation of NNs with a different set of transfer functions. While NNs typically only contain a certain type of sigmoid or radial basis functions, CPPNs can comprise a mixed configuration of various types of functions. Moreover, its choice of transfer functions can be biased toward specific types of patterns and regularities [27]. For example, periodic functions such as sine produce segmented patterns with repetitions, while symmetric functions such as Gaussian produce symmetric patterns; linear functions can produce either linear or fractal-like patterns.

Deep neural networks are good at learning non-linearity and low-level vision features [28], such as the feature vector learnt from each image block for deciding block similarities. In 1957, Kolmogorov [29] proved a theorem stating that, any continuous multivariate function can be exactly represented by superpositioning several continuous univariate functions. To make it easier to understand, we present the Kahane's representation [30] of this Kolmogorov theorem below.

$$
f\left(x_{1}, x_{2}, \ldots, x_{n}\right)=\sum_{q=1}^{2 n+1} g\left(\sum_{p=1}^{n} \lambda_{p} h_{q}\left(x_{p}\right)\right)
$$

Nielsen [31] pointed out that, this theorem can be interpreted in the context of NN. Specifically, any continuous multivariate function is equivalent to an $\mathrm{NN}$ with two hidden layers (i.e. four-layer $\mathrm{NN}$ ) whose transfer functions are all continuous univariate functions. Our understanding of this theorem is that, it essentially breaks down the complexity of the function $f(\cdot)$ to be learnt by eliminating the direct correlation terms among different input variables, and complement such correlation at the 2 nd hidden layer in an approximate way. However, Kolmogorov's proof is not 
completely algorithmic as it does not describe how to derive $g(\cdot)$ and $h_{d}(\cdot)$ from $f(\cdot)$. To solve this, many algorithmic solutions have been proposed, such as the one by Kurkova [32].

In the convolutional layer of the CPPNs, each neuron in this layer is connected to a small region of the input neurons, say, for example, a $5 \times 5$ region, corresponding to 25 input pixels, which is called the local receptive field for the hidden neuron. Due to the convolutional essence of this layer, we use the same weights and bias for each of the hidden neurons. The overall connection topology and examples are shown in Figure 3.

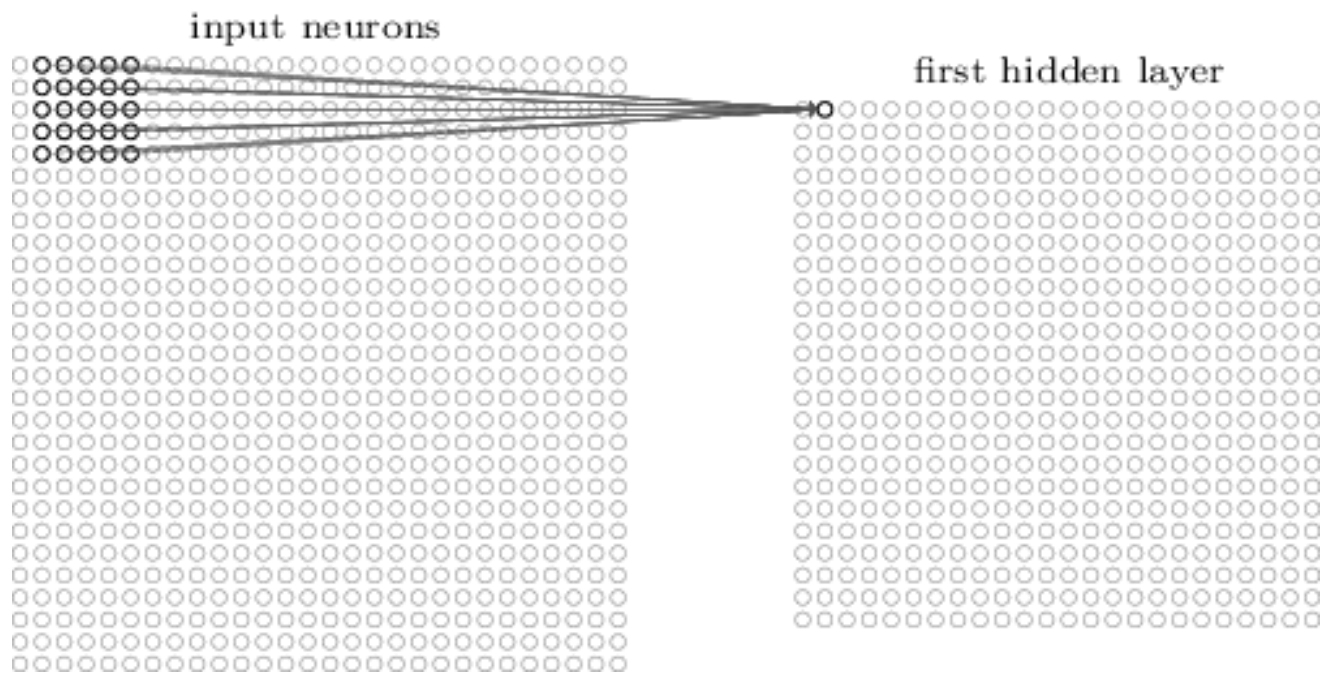

Figure 3. The connection topology between the input layer and the convolutional layer

Figure 4 plots the topology of feed-forward back propagation NNs, which is specifically designed for feature vector forgery detections. The input, the 1 st hidden, the 2 nd hidden and the output layers has 50, 121, 3 and 1 neuron respectively (i.e. a 50-121-3-1 topology *). For the sake of adjustability and flexibilities, the 1st hidden layer has multiple transfer functions, each of which is assigned a specific number of neurons as listed in Table 1. Here, $f(x)=x$ is a linear filter, and $f(x)=\exp (x)$ is to cancel out the logarithms of pixel values. Note that the selected 9 transfer functions are commonly used to approximate general high-order functions. The 2nd hidden layer has 3 transfer functions, namely, $f(x)=x, \log \operatorname{Sig}(x)$ and radialBasis $(x)$, for maintaining both linearity and nonlinearity passed in from the 1 st layer. Finally, the output layer has a linear transfer function. Note that the reason for having a linear transfer function at every layer is to leave a passageway for imitating purely linear feature models.

\footnotetext{
* Other NN topologies with similar size only lead to a slight performance difference. Investigation of the optimal topology is a pure machine learning problem, which is out of the scope of this work.
} 
Table 1. Transfer functions in the 1st hidden layer

\begin{tabular}{|c|c|}
\hline Transfer function definition & $\begin{array}{c}\text { No. of neurons } \\
\text { assigned }\end{array}$ \\
\hline $\operatorname{tanSig}(x)=2 /(1+\exp (-2 x))-1$ & 16 \\
\hline $\log \operatorname{Sig}(x)=1 /(1+\exp (-x))$ & 16 \\
\hline doubleSig$(x)=\exp (-x) /(1+\exp (-x))^{2}$ & 16 \\
\hline doubleLogSig $(x)=\operatorname{sgn}^{\dagger}(x)\left(1-\exp \left(-x^{2}\right)\right)$ & 16 \\
\hline$f(x)=\cos (x)$ & 16 \\
\hline $\operatorname{radialBasis}(x)=\exp \left(-x^{2}\right)$ & 16 \\
\hline$f(x)=1-|x|,-1 \leq x \leq 1, f(x)=0$ otherwise & 16 \\
\hline$f(x)=\exp (x)$ & 8 \\
\hline$f(x)=x$ & 1 \\
\hline
\end{tabular}

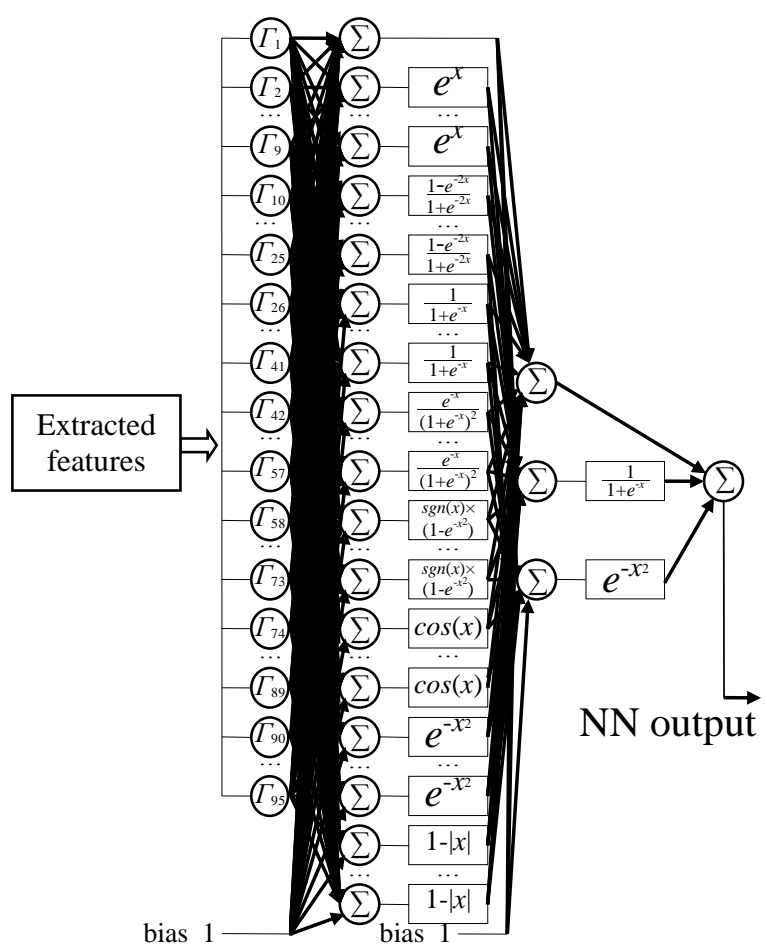

Figure 4. Topology of the proposed CPNNs, where $\Gamma_{1}, \Gamma_{2}, \Gamma_{3}, \ldots, \Gamma_{95}$ represents feature maps, which constitutes the convolutional layer of a CPNN.

Before training, both input and target values to NNs are proportionally scaled so that they correspond to the sensitive region of the transfer function. We use the stochastic gradient descent method proposed in [33] as the training algorithm.

\footnotetext{
${ }^{\dagger} \operatorname{sgn}(x)$ represents the sign function. $\operatorname{sgn}(x)=0$ for $x=0 ; \operatorname{sgn}(x)=\mathrm{x} /|\mathrm{x}|$ for $x \neq 0$.
} 


\section{Experimental Results and Analysis}

All the experiments discussed here are carried out with Visual Studio 2013 written using C++ code running on a $2.4 \mathrm{GHz}$ quad core ( 8 threads with hyper-threading) Intel Core i7-5500U CPU processor with $8 \mathrm{G}$ memory under 64bit mode. The tampered images are created by an open source image editing program, GIMP, using images of size 512x512 from the publicly available CoMoFoD database[18].

The success of a forgery detection method is measured with a normalized metric called accuracy ratio $a$ in the [0-1] range. An accuracy ratio of 1 corresponds to the correct detection of all copied and pasted regions. Accuracy ratio decreases if the method marks regions not forged as forged. Let ground truth copied and pasted regions in a fake image be $T_{1}$ and $T_{2}$, whereas copied and pasted regions detected by the algorithm be $M_{1}$ and $M_{2}$ respectively. The accuracy ratio of the algorithm is calculated by using the following equations:

$$
\begin{gathered}
a=\frac{\left|T_{1} \cap M_{1}\right|+\left|T_{2} \cap M_{2}\right|}{\left|T_{1}\right|+\left|T_{2}\right|} \\
f=\frac{\left|T_{1} \cup M_{1}\right|+\left|T_{2} \cup M_{2}\right|}{\left|T_{1}\right|+\left|T_{2}\right|}-a
\end{gathered}
$$

False negative ratio, $f$ is also used during experiments to have a measure of the regions detected as forged but actually they are not. The success of the forgery detection algorithm improves as the value of $f$ approaches to zero.

Proposed method is evaluated using the following set of experiments. Each experiment gives an idea about the robustness of the method and a comparison with similar works:

(1) Our first set of experiments indicates forged regions visually and reports accuracy ratios of the method when post-processing operations are not applied after forgery. Forged regions are chosen to be regular and non-regular shape. First experiment also evaluates the effectiveness of the method when the forged image has more than one copied and pasted regions.

(2) Our second set of experiments indicates robustness of the method when the doctored image is post processed using some methods such as JPEG compression, Gaussian blurring and Additive White Gaussian Noise (AWGN).

(3) Our third set of experiments compares the method with similar detection methods reported in the literature. 


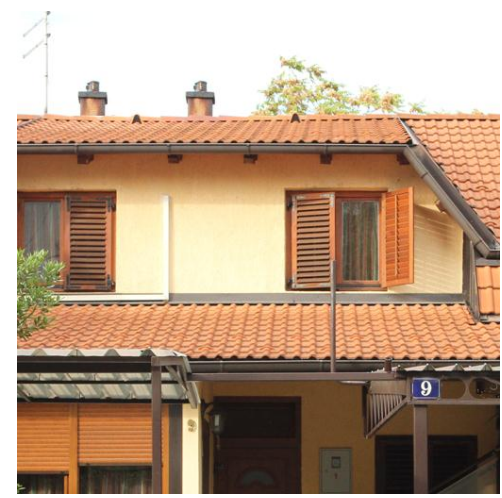

(a)

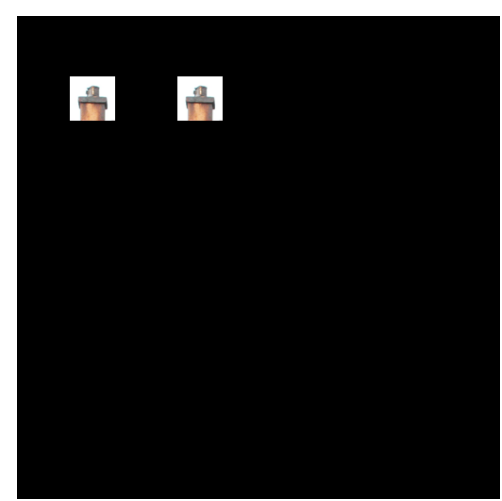

(d)

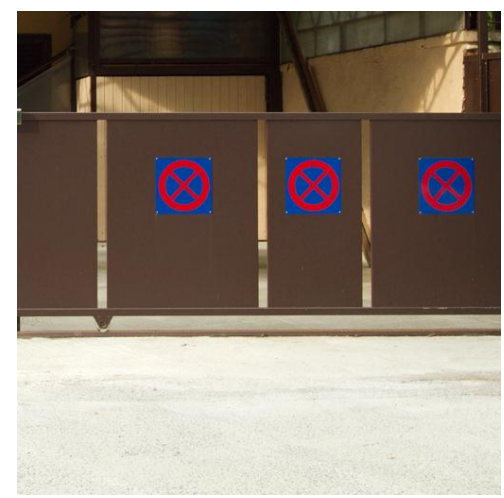

(b)

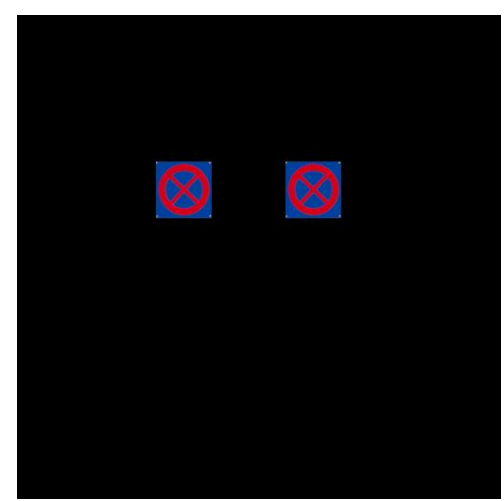

(e)

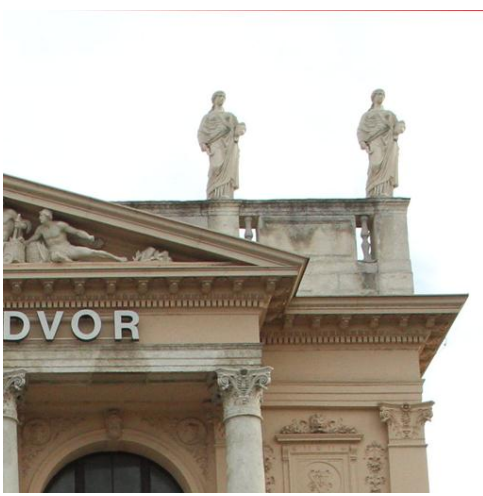

(c)

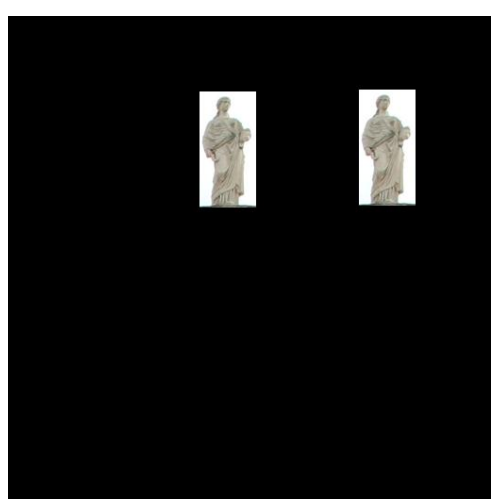

(f)

Figure 5. (a) (b) (c) Forged images with regular regions of size 48x48, 60x60 and 60x120 respectively (d) (e) (f) Only the copied and pasted regions detected are displayed and other parts of the images are masked as black.

In the first experiment, three scenarios are realized to show the effectiveness and accuracy of the method under the regular, non-regular and multiple copy-move forgery operations. The forged images are created using GIMP without any post processing operations. Two color images of size $512 \times 512$ are doctored without any post processing operation. Figure 5(a), 5(b) and 5(c) show the regular copy-move forgery operation. Rectangular shaped regular regions of sizes 48x48, 60x60 and 60x120 pixels are copied and pasted as indicated by the masks shown in Figure 5(d), 5(e) and 5(f). Figure 5(d), 5(e) and 5(f) are the results of the forgery detection algorithm where only the copied and pasted regions detected are displayed and other parts of the images are masked as black. Accuracy ratios of the algorithm for these test images are approximately 1 as can be seen in the figure. This means that copied and pasted regions are almost determined correctly and accurately by the our proposed method.

Figure 6 shows the non-regular copy-move forgery operation. Figure 6(a), 6(b) and 6(c) show the non-regular copy-move forgery operation, in which non-regular shaped regions are copied and pasted as indicated by the masks shown in Figure 6(d), 6(e) and 6(f). Figure 6(d), 6(e) and 6(f) are 
the results of the forgery detection algorithm where only the copied and pasted regions detected are displayed and other parts of the images are masked as black. Our proposed method yields accuracy ratios higher than 0.95 even though doctored images have non-regular shaped forged regions.

Our proposed method can also detect doctored regions on the image even though the image has multiple copied and pasted regions as can be seen in Figure 7. Figure 7(d), Figure 7(e) and 7(f) show the detection result under multiple copy move forgery operation. Figure 7 indicates that, our proposed method also yields high accuracy ratio even if the image has multiple copied and pasted regions.

The experiments presented in Figure 5, Figure 6 and Figure 7 indicate that our proposed method gives satisfactory results when the doctored image has regular and non-regular forged regions or multiple copied and pasted regions.

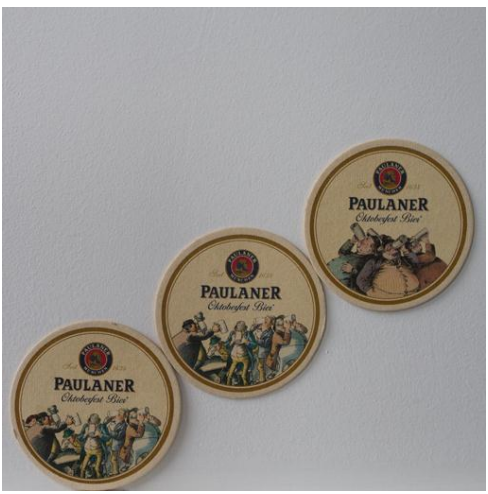

(a)

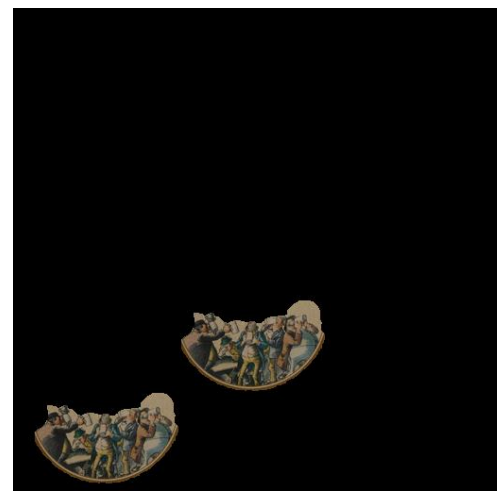

(d)

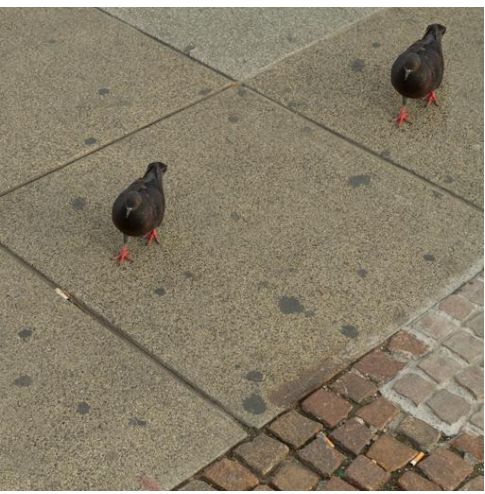

(b)

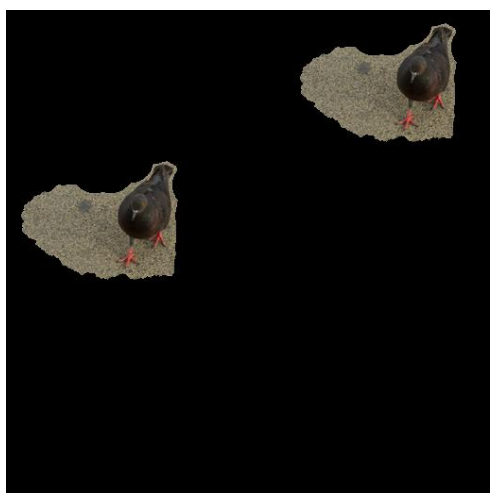

(e)

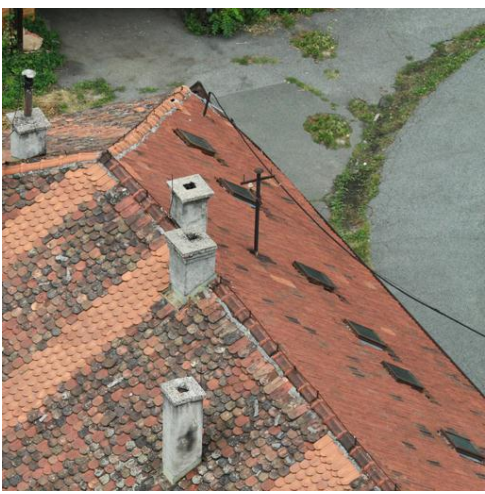

(c)

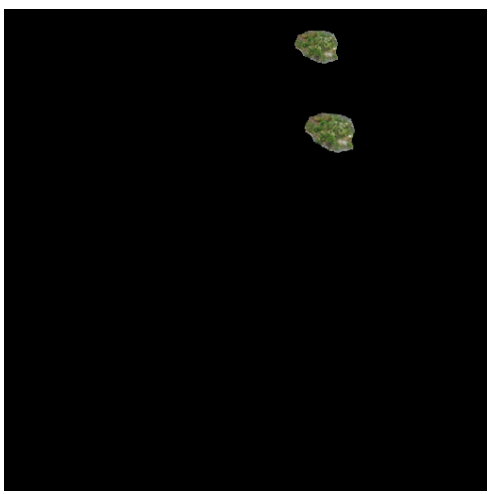

(f)

Figure 6. (a) (b) (c) Forged images with non-regular regions (d) (e) (f) Only the copied and pasted regions detected are displayed and other parts of the images are masked as black. 


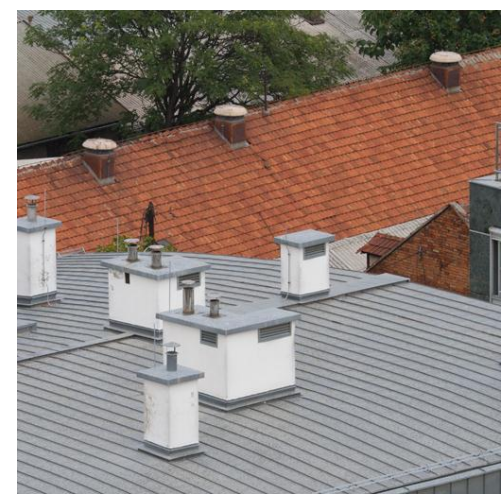

(a)

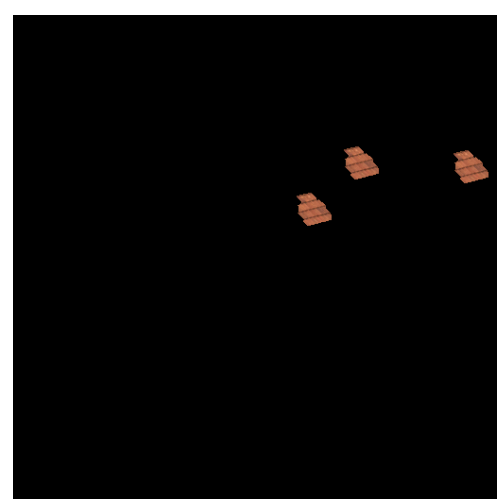

(d)

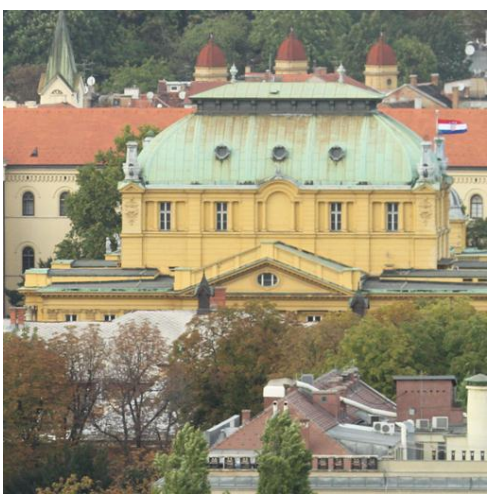

(b)

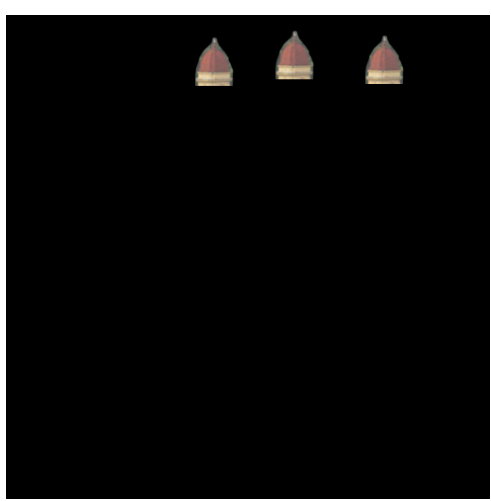

(e)

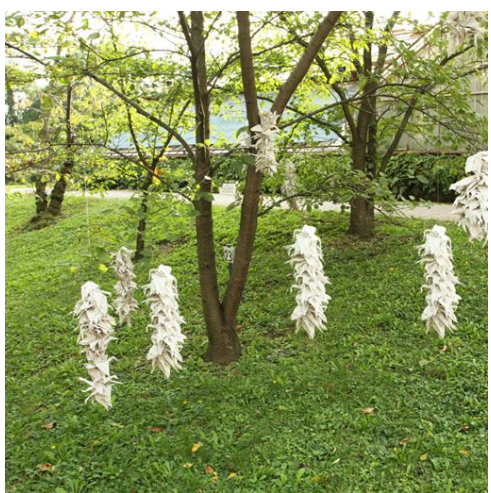

(c)

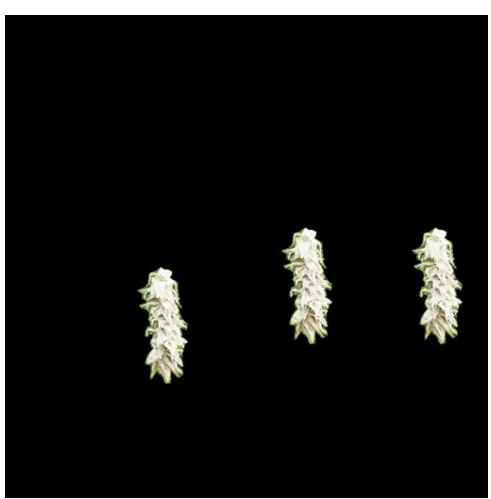

(f)

Figure 7. (a) (b) (c) Forged images with multiple non-regular regions (d) (e) (f) Only the copied and pasted regions detected are displayed and other parts of the images are masked as black.

In the second set of experiments, our aim is to test the effectiveness of the method when the doctored images have gone some post processing operations. Image editing experts in making digital forgeries use post-processing operations after forgery to hide visible clues. JPEG compression, Gaussian blurring or additive white Gaussian noises are the most widely used operations either alone or together to hide traces of forgery. These operations are performed on forged images with multiple copied and pasted regions to show the robustness of the method. In order to test robustness against Additive White Gaussian Noise with SNR $20 \mathrm{~dB}$ and $27 \mathrm{~dB}$, the image to be detected are shown in Figure 8(a) and 8(d) respectively. Accuracy ratio of the method improves as the value of SNR increases. The method yields approximately 0.91 accuracy ratio even for $27 \mathrm{~dB}$ SNR. Another experiment uses Gaussian blurring operation on the forged image, the forged image is post processed with window size $5 \times 5$ for both $\sigma=2$ and $\sigma=4$. Figure $8($ b) and Figure $8($ e) illustrate the images to be detected. The accuracy ratios are approximately 0.92 and 0.90 respectively for these two post processed images. Robustness of the method against JPEG compression is also tested in this section. Forged image is saved with quality factor 75 and 85 respectively. Figure 8(c) and 8(f) shows the image to be detected. Accuracy ratios of the detected regions are also reported below the images. The 
method has approximately 0.88 accuracy ratios when the forged image is saved with quality factor 75 . Robustness of the method against the JPEG compression is satisfactory as indicated in the results.

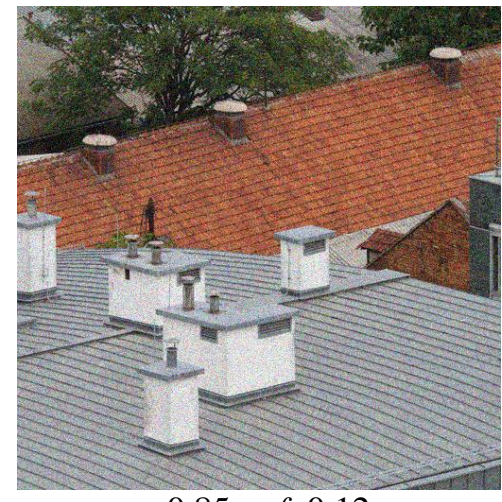

$p=0.85, \quad f=0.12$

(a)

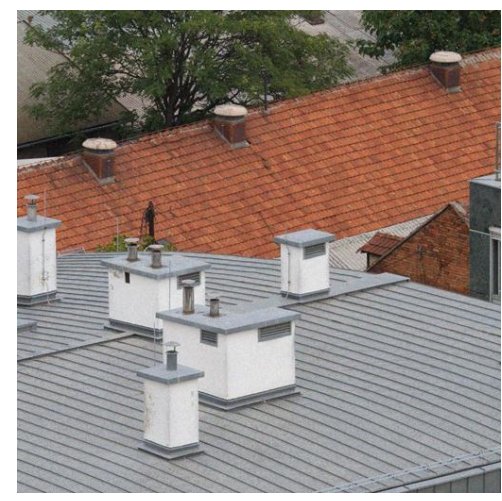

$p=0.91, f=0.08$

(d)

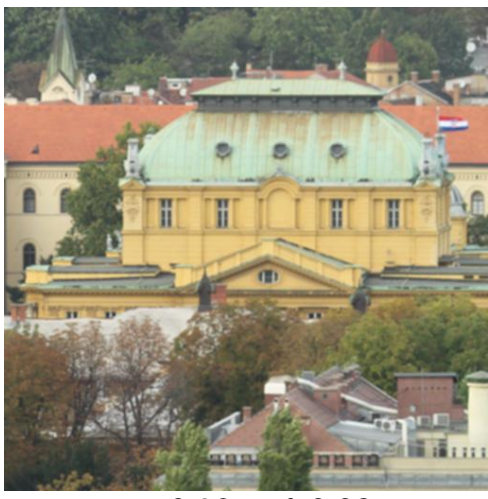

$p=0.92, \quad f=0.08$

(b)

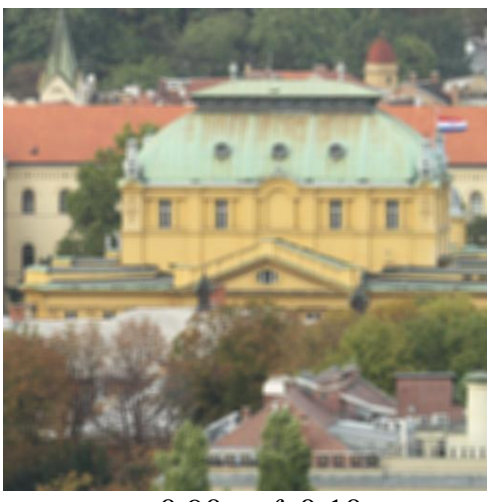

$p=0.90, \quad f=0.10$

(e)

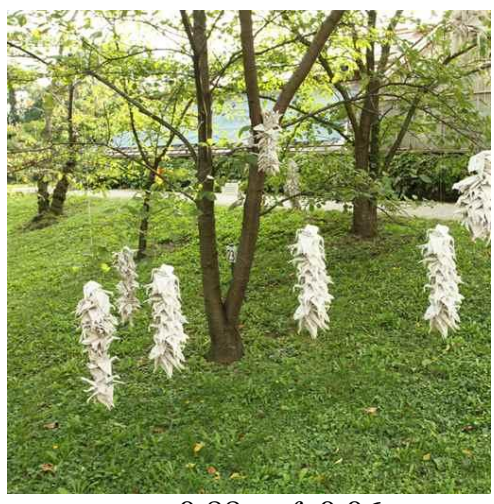

$p=0.88, \quad f=0.06$

(c)

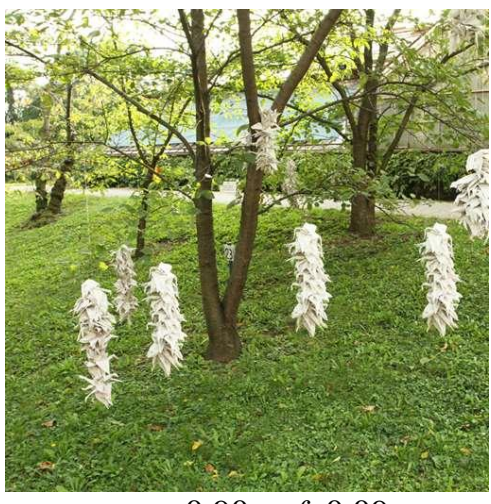

$$
p=0.90, \quad f=0.09
$$

(f)

Figure 8. Forged images with multiple non-regular regions subject to (a) additive white Gaussian noise (SNR $=20 \mathrm{~dB}$ ), (b) $5 \times 5$ Gaussian Blurring with $\sigma=4$, (c) JPEG compression with quality factor 75, (d) additive white Gaussian noise ( $\mathrm{SNR}=27 \mathrm{~dB}$ ), (e) $5 \times 5$ Gaussian Blurring with $\sigma=2$, (f) JPEG compression with quality factor $85 \mathrm{~dB}$. The accuracy ratios and false negative ratios of the detected regions are marked below the images.

We also quantitatively evaluate the proposed method for the robustness under various post processing operations. The test scenario given in [7] is used for the sake of having a fair comparison. 100 original images are selected randomly from the CoMoFod dataset to generate doctored images. Square regions of sizes $32 \times 32,48 \times 48,64 \times 64$ and $96 \times 96$ pixels from randomly selected locations are pasted onto non-overlapping regions to generate 400 tampered images. Tampered images are also post processed with commonly used white Gaussian noise, JPEG compression, Gamma correction, and median filtering post processing operations to show the robustness of our the proposed method. 


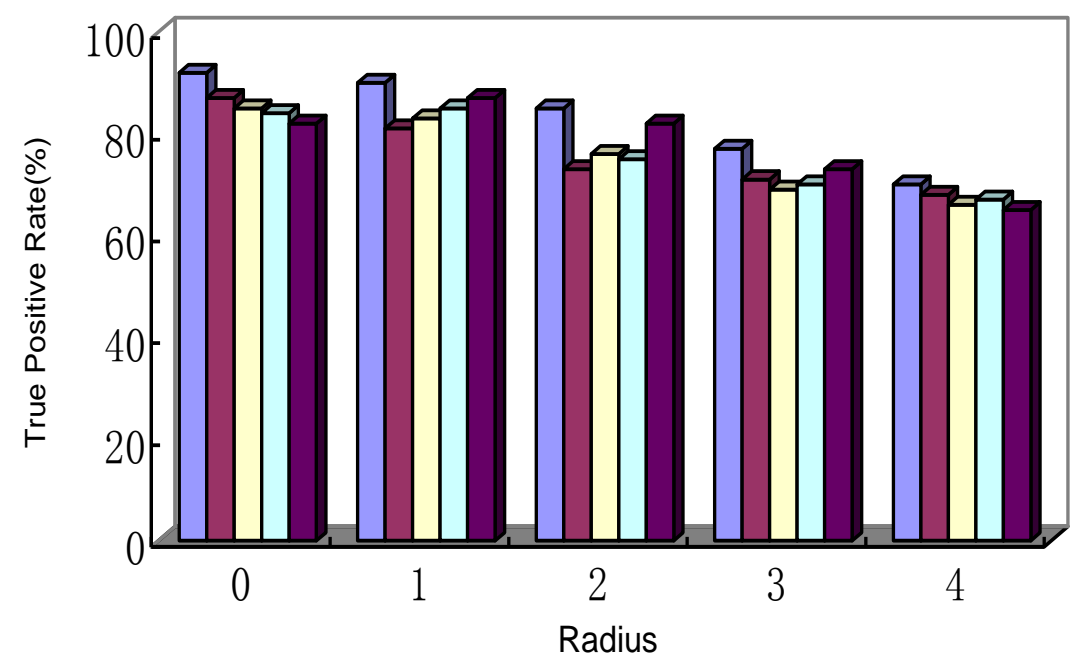

\begin{tabular}{|lllll|}
\hline $32 \times 32$ & $\square 48 \times 48$ & $\square 64 \times 64 \quad \square 80 \times 80$ & $\square 96 \times 96$
\end{tabular}

(a)

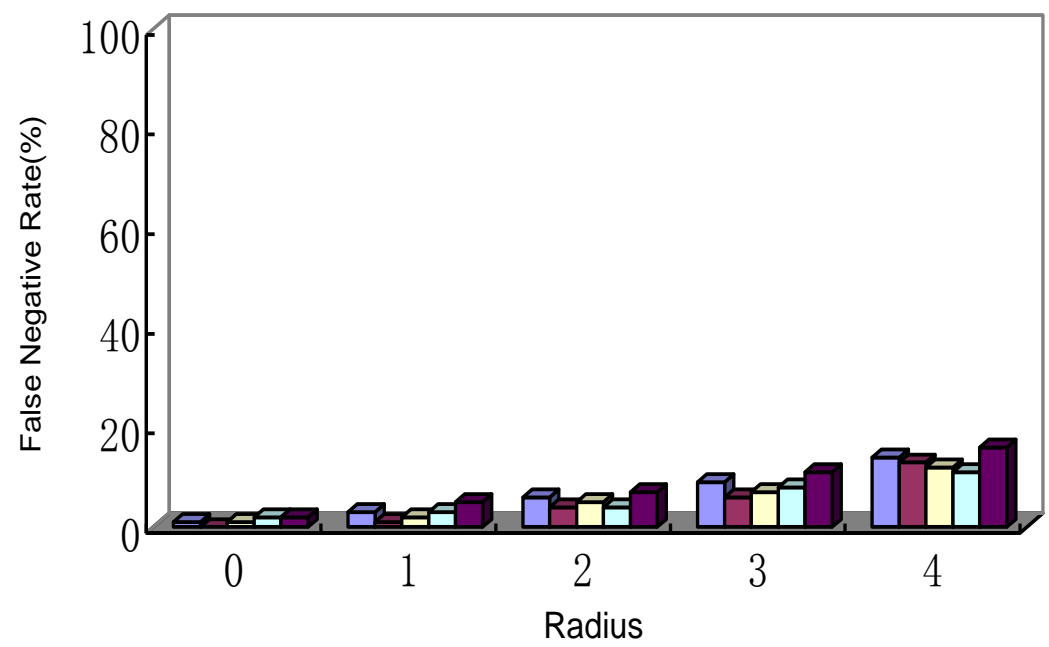

$\square 32 \times 32 \square 48 \times 48 \quad \square 64 \times 64 \quad \square 80 \times 80 \quad \square 96 \times 96$

(b)

Figure 9. (a) True positive rate, and (b) false negative rate of the proposed method with the post processing operation median filtering with kernel radius $0,1,2,3$ and 4 .

The recognition true positive and false negative rates are summarized in Figure 9 for the median filtering post processing operation with different parameters. The recognition true positive rate is higher than 0.85 even if the forged image has been $5 \times 5$ median filtered (with median filtering with kernel radius 2) with small tampering image patch size.

The forgery detection results of the proposed method with white Gaussian noise are given in Figure 10. The performance of the method improves as region size decreases. The method has accuracy ratios higher than 0.836 even if it is distorted with $33 \mathrm{~dB}$ noise signal. 
JPEG compression is also implemented with various quality factors to test the robustness of the method as given in Figure 11. Performance of the method increases while the quality factor is increased. It is noted that, the algorithm marks forged regions for quality factor 80 with 0.89 true positive rate. It is seen that the proposed method can detect forgery operation with high performance in the case of the slight compression.

Figure 12 shows the affect of gamma correction on the performance of the proposed method. It can be seen that, the performance of the proposed method steadily and robustly declines as the gamma value goes far away from one.

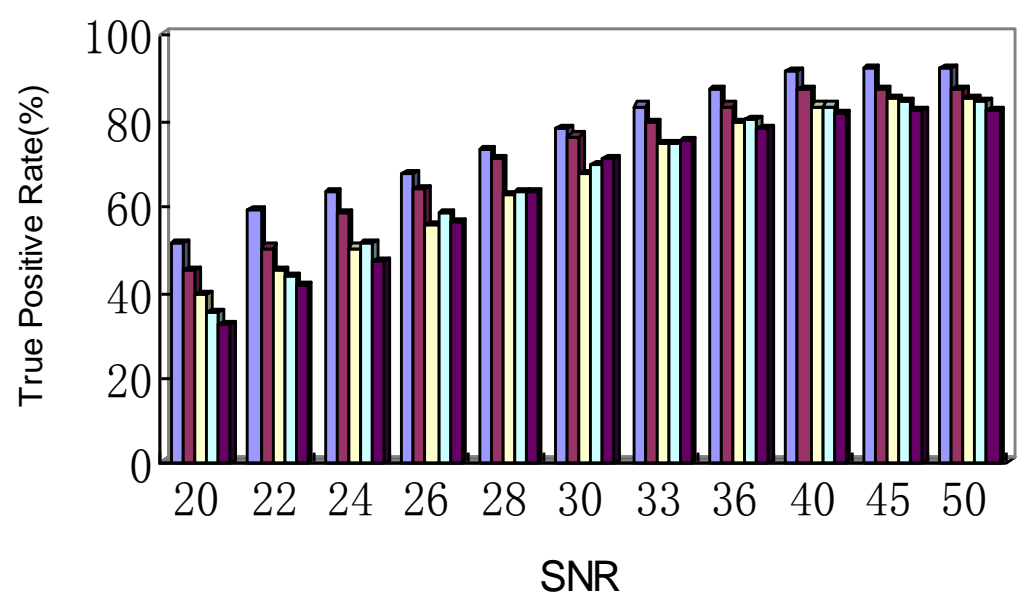

\begin{tabular}{|lllll|}
\hline$\square 32 \times 32 \quad \square 48 \times 48 \quad \square 64 \times 64 \quad \square 80 \times 80 \quad \square 96 \times 96$ \\
\hline
\end{tabular}

(a)

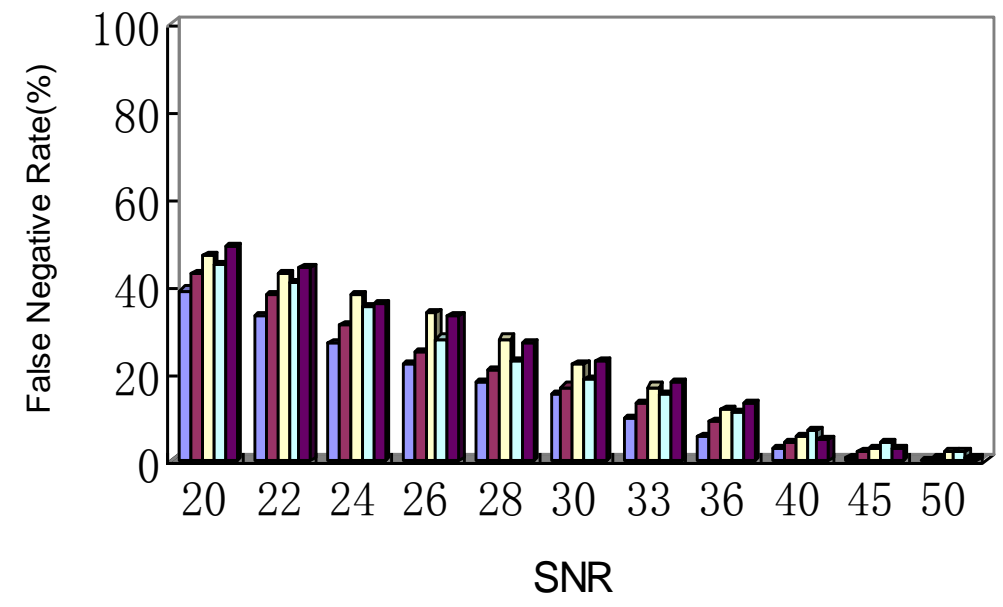

\section{$\square 32 \times 32 \square 48 \times 48 \quad \square 64 \times 64 \quad \square 80 \times 80 \quad \square 96 \times 96$}

(b)

Figure 10. (a) True positive rate, and (b) false negative rate of the proposed method under white Gaussian noise with SNR (Signal-to-Noise Ratio) of 20, 22, 24, 26, 28, 30, 33, 36, 40, 45 and 50. 


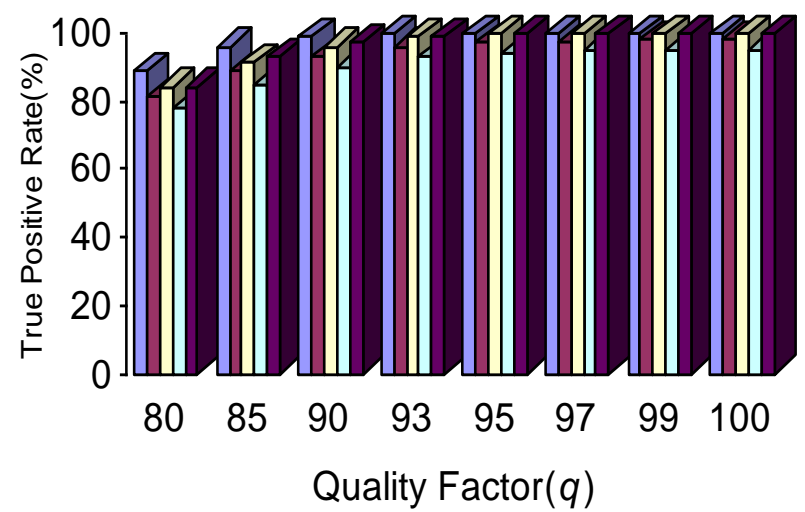

\section{$32 \times 32 \square 48 \times 48 \square 64 \times 64 \square 80 \times 80 \square 96 \times 96$}

(a)

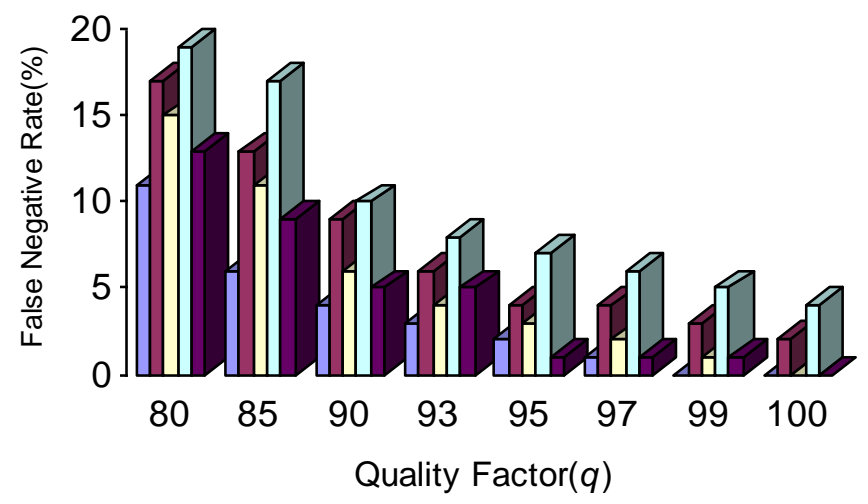

$32 \times 32 \square 48 \times 48 \square 64 \times 64 \square 80 \times 80 \square 96 \times 96$

(b)

Figure 11. (a) True positive rate, and (b) false negative rate of the proposed method under JPEG compression with quality factor $80,85,90,93,97,99$ and 100 .

The third set of experiments compares the proposed method with three methods reported in the literature. DCT based methods are chosen for comparison since our proposed method uses the CLD for feature extraction. DCT constitutes a base platform for calculation of the CLD. The robust DCT method [5], the improved DCT method [6], and the DCT-SVD method [7] are used for performance comparison purpose. 150 test images that have $48 \times 48$ pixel forged regions are selected from the test database. True positive and false negative ratios are adopted as the performance metrics. Postprocessing operations are applied on the test images. Modified and post processed forged images with white Gaussian noise are used first, and the result is shown in Figure 13(a). The true positive 
rate of all methods increases as the noise level decrease (i.e. the SNR increases). Our proposed method gives higher recognition rate compared to other methods. Similarly Modified and post processed forged images with JPEG compression, gamma correction, and median filtering are also used to test the four methods to compare their performance and robustness to post processing corruptions. It is seen from Figure 13 that, all four methods are robust to all these post processing operations, and obviously our proposed method has the highest performance overall.

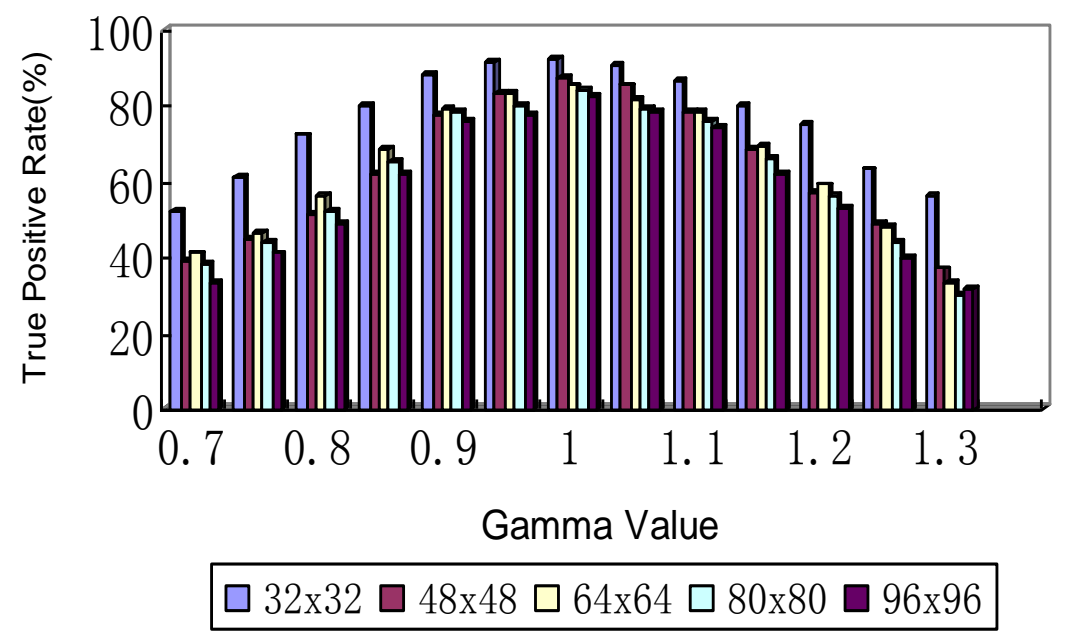

(a)

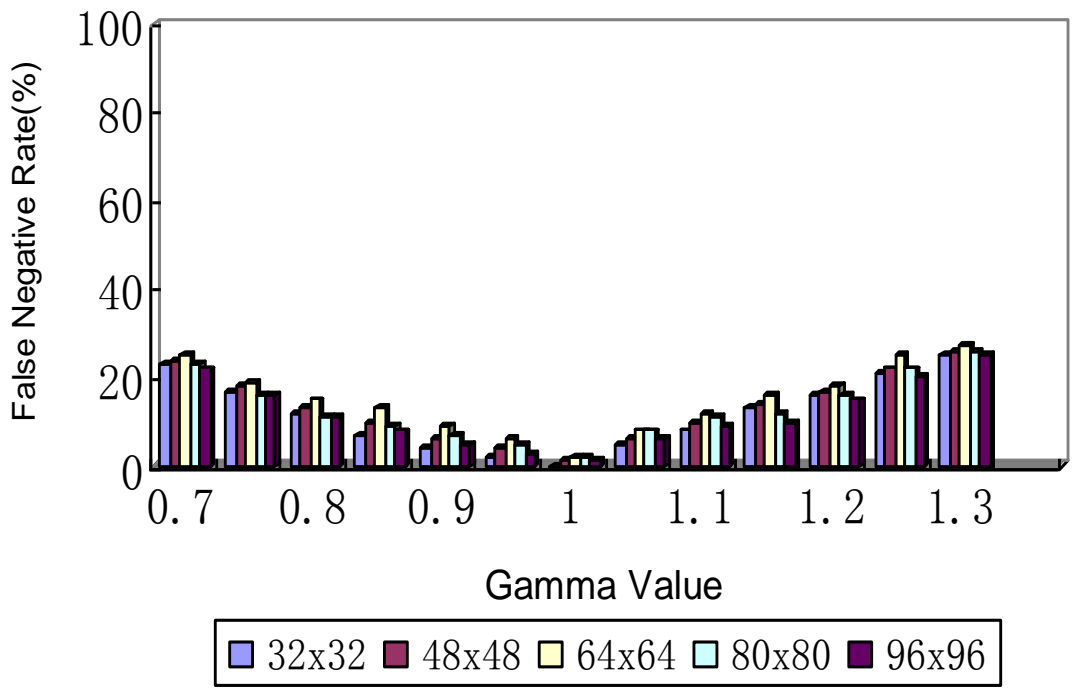

(b)

Figure 12. (a) True positive rate, and (b) false negative rate of the proposed method under gamma correction with gamma value of 20, 22, 24, 26, 28, 30, 33, 36, 40, 45 and 50. 


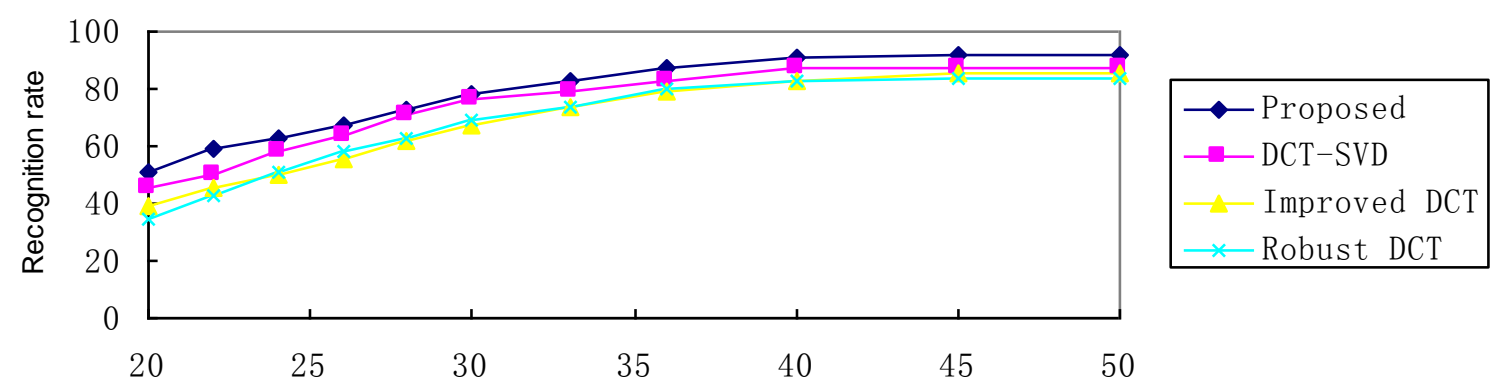

(a) $\operatorname{SNR}(\mathrm{db})$

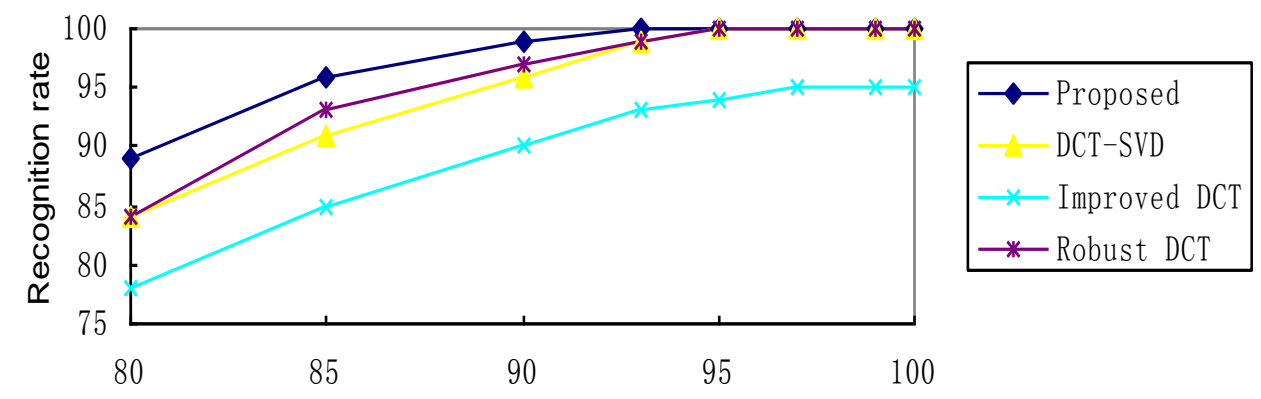

(b) JPEG quality

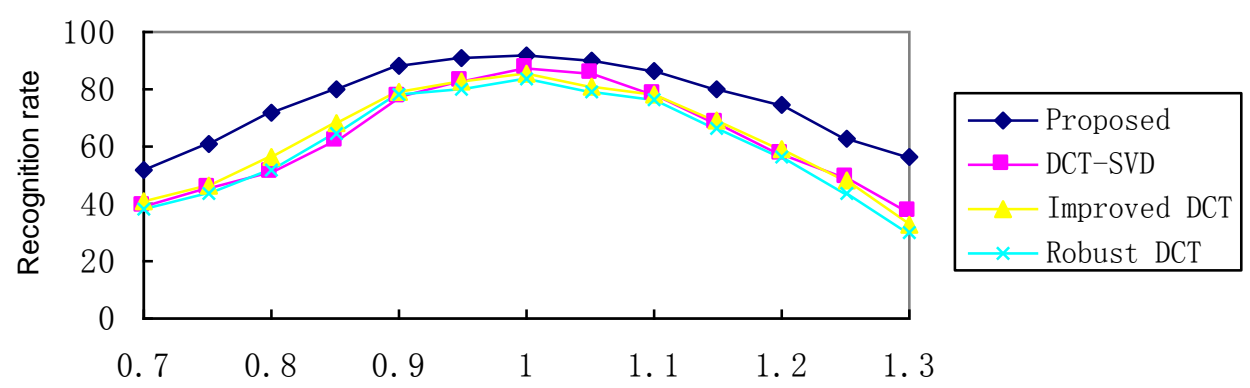

(c) Gamma Value

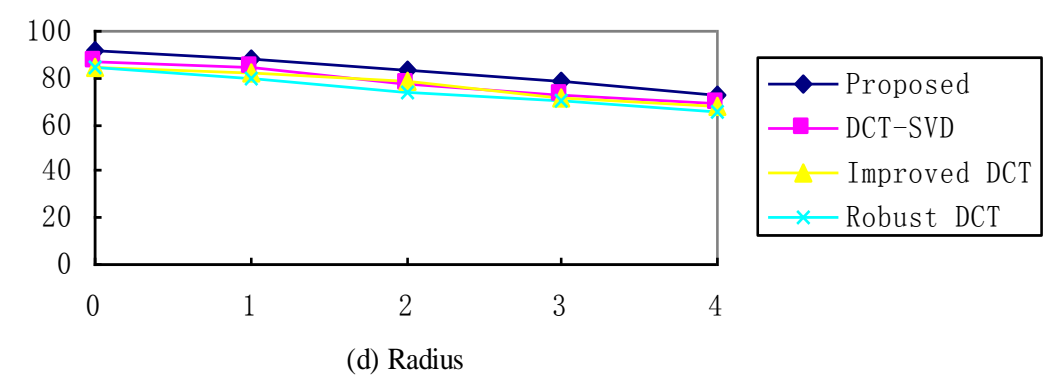

Figure 13. (a-d) Recognition rates for different methods as a function of SNR (Signal-to-Noise Ratio) of white Gaussian noise, JPEG quality, the $\gamma$ value of gamma correction, radius of median filter respectively. Each data point corresponds to a recognition rate over 150 images. 


\section{Conclusion}

In this paper, we proposed a digital image forgery detection method based on a variety of image descriptors and feature, such as the color moments, color layout descriptors, color and edge directivity descriptor, fuzzy color and texture histogram, scalable color descriptor, and edge histogram descriptor. The proposed method ensures the robustness against some post processing operations such as white Gaussian noise, blurring, JPEG compression or gamma correction. The proposed method yields high accuracy ratio even when the test image is corrupted with moderate noise. The proposed method can also perform the forgery detection with satisfactory accuracy ratios when the test image is quite blurry. And the propose method is with acceptable detection ability when the forged image is with low JPEG compression quality facto. The experimental results also indicate than our proposed method has higher accuracy ratios and lower false negative values compared to other DCT based methods in the existing literature.

Our next research plan is to improve the forgery detection method using feature extractors that are robust to rotation, scaling and translation attacks.

\section{References}

1. Shih-Fu Chang, Thomas Sikora, and Atul Purl. Overview of the mpeg-7 standard. IEEE Transactions on Circuits and Systems for Video Technology, 11(6):688-695, 2001.

2. J. Fridrich, D. Soukal, and J. Luka' s, Detection of copy-move forgery in digital images, in: Proceedings of DFRWS 2003, Cleveland, OH, USA, 2003.

3. X. Wang, X. Zhang, Z. Li, and S. Wang, A DWT-DCT based passive forensics method for copymove attacks, in: 2011 Third International Conference on Multimedia Information Networking and Security, 2011, 304-308.

4. J. Hu, H. Zhang, Q. Gao, and H. Huang, An improved lexicographical sort algorithm of copymove forgery detection, in: Proceedings - 2nd International Conference on Networking and Distributed Computing, ICNDC 2011, (2011), pp. 23-27.

5. Y. Huang, W. Lu, W. Sun, and D. Long, Improved DCT-based detection of copy-move forgery in images, Forensic Sci. Int. 3 (2011) 178-184.

6. Y. Cao, T. Gao, L. Fan, and Q. Yang, A robust detection algorithm for copy-move forgery in digital images, Forensic Sci. Int. 214(2012) 33-43.

7. J. Zhao, and J. Guo, Passive forensics for copy-move image forgery using a method based on DCT and SVD, Forensic Sci. Int. 233(2013) 158-166.

8. A.N. Myrna, M.G. Venkateshmurthy, and C.G. Patil, Detection of Region Duplication Forgery in Digital Images Using Wavelets and Log-Polar Mapping, in: Conference on Computational Intelligence and Multimedia Applications, 2007, International Conference, vol. 3, 2007, 371-377. 
9. S. Bayram, H.T. Sencar, and N. Memon, An efficient and robust method for detecting copy-move forgery, in: Proceedings of IEEE International Conference on Acoustics, Speech and Signal Processing, 2009, pp. 1053-1056.

10. A.C. Popescu, and H. Farid, Exposing digital forgeries by detecting duplicated image regions, no. TR2004-515, 2004, 1-11.

11. M. Zimba, and S. Xingming, DWT-PCA (EVD) based copy-move image forgery detection, Int. J. Digital Content Technol. Appl. 5 (1) (2011) 251-258.

12. G. Li, Q. Wu, D. Tu, and S. Sun, A sorted neighborhood approach for detecting duplicated regions in image forgeries based on DWT and SVD, in: Multimedia and Expo, 2007 IEEE International Conference, 2007, 1750-1753.

13. Z. Ting, and W. Rang-Ding, Copy-move forgery detection based on SVD in digital image, in: Proceedings of the 2009 2nd International Congress on Image and Signal Processing, CISP'09, 2009. 14. W. Luo, J. Huang, and G. Qiu, Robust detection of region duplication forgery in digital images, in: International Conference on Pattern Recognition, (4) 2006 746-749.

15. B. Mahdian, and S. Saic, Detection of copy move forgery using a method based on blur moment invariants, Forensic Science International, 171(2007), 180-189.

16. E. Kasutani, and A. Yamada, The MPEG-7 color layout descriptor: a compact image feature description for high-speed image/video segment retrieval, 2001, 674-677.

17. M. Stricker, and M. Orengo, "Similarity of color images", In Proceedings of SPIE Conference on Storage and Retrieval for Image and Video Databases, 1995, 381-392, San Jose, USA.

18. CoMoFoD database, available at: http://www.vcl.fer.hr/comofod

19. Donald E Gustafson, and William C Kessel, Fuzzy clustering with a fuzzy covariance matrix. In IEEE Conference on Decision and Control, volume 17, pages 761-766, 1978.

20. Savvas A. Chatzichristofis, and Yiannis S. Boutalis. CEDD: Color and edge directivity descriptor: A compact descriptor for image indexing and retrieval. In Lecture notes in Computer Sciences, volume 5008, pages 312-322, 2008.

21. Savvas A. Chatzichristofis, and Yiannis S. Boutalis. FCTH: Fuzzy color and texture histogram: A low level feature for accurate image retrieval. In Proceedings of the 9th International Workshop on Image Analysis for Multimedia Interactive Service, pages 191-196, 2008.

22. R. Balasubramani, and V.Kannan, Efficient use of MPEG-7 Color Layout and Edge Histogram Descriptors in CBIR Systems. Global Journal of Computer Science and Technology, 9(4): 157-163, 2009.

23. Y.J. Long and Y.Z. Huang, Image based source camera identification using demosaicking, IEEE 8th Workshop on Multimedia Signal Processing, pp.419-424, 2006. 
24. Y.Z. Huang and Y.J. Long, Super-resolution using neural networks based on the optimal recovery theory, IEEE 16th Workshop on Machine Learning for Signal Processing, pp.465-470, 2006.

25. Y.Z. Huang and Y.J. Long, Demosaicking recognition with applications in digital photo authentication based on a quadratic pixel correlation model, In Proceedings of IEEE Conference on Computer Vision and Pattern Recognition 2008, pp.1-8.

26. Y.Z. Huang and N. Fan, Learning from interpolated images using neural networks for digital forensics, In Proceedings of IEEE Conference on Computer Vision and Pattern Recognition 2010, pp.177-182.

27. Q.Y. Jin, Y.Z. Huang and N. Fan, Learning images using compositional pattern-producing neural networks for source camera identification and digital demographic diagnosis, Pattern Recognition Letters, vol.33, no.4, pp.381-396, 2012.

28. J. Yosinski, J. Clune, Y. Bengio, and H. Lipson, How transferable are features in deep neural networks? In Proceedings of Advances in Neural Information Processing Systems 2014, pp.33203328.

29. A.N. Kolmogorov, On the representation of continuous functions of several variables by superposition of continuous functions of one variable and addition, Doklady Akademii Nauk SSSR, 114: 953-956, 1957.

30. J.P. Kahane, Sur le théorème de superposition de Kolmogorov, Journal of Approximation Theory, 13(3): 229-234, 1975.

31. R.H. Nielsen, Kolmogorov's mapping neural network existence theorem, Proceedings of IEEE International Conference on Neural Networks 1987, pp.11-14.

32. V. Kurkova, Kolmogorov's theorem and multilayer neural networks, Neural Networks, 5(3): 501506, 1992.

33. R. Johnson, and T. Zhang, Accelerating stochastic gradient descent using predictive variance reduction, In Proceedings of Advances in Neural Information Processing Systems 2013, pp.315-323. 